partnerships. Their honest and frank feedback was essential to broaching sensitive topics related to partnership development, and to identify realistic and practical solutions. We also thank all members of the planning committee and our colleagues in the Community Engagement Program for their work on bringing together community and academic members for this retreat. This project was supported by grant number UL1TR002240 from the National Center for Advancing Translational Sciences (NCATS).

\section{Developing a REDCap Database to Understand Partnership Cultivation Efforts}

Grisel M. Robles-Schrader ${ }^{1}$, Gina Curry, Josefina Serrato, Jen Brown and Keith A Herzog

${ }^{1}$ Northwestern University

OBJECTIVES/SPECIFIC AIMS:.Outline the development and purpose of the partnership brokering database in REDCap. Provide an overview of the tool and how it works. Discuss how this tool facilitates partnership-brokering activities and discuss plans for future use METHODS/STUDY POPULATION: Research Electronic Data Capture (REDCap) is a secure, web-based application developed at Vanderbilt University to assist with systematic data management of small and medium sized projects. CCH utilized REDCap to build a custom data management warehouse entitled the Partnership Brokering Tool. Information compiled in various formats (handwritten notes, spreadsheets, etc.) over the past 10 years by CCH staff, was then systematically organized and entered into the Partnership Brokering Tool. The tool captures information such as individual contact information, organizational affiliation (academic, community, faith, government etc.), research interests (35 categories asthma, diabetes, heart disease, etc.), communities of foci (children, elderly, LGBTQ, ethnicity, etc.), and target geographic community served (Chicago north, south, suburban, Illinois, etc.). RESULTS/ ANTICIPATED RESULTS: Data was compiled on 451 community groups and organizations and 77 partners in academia thus far. Community organizations represent a range of community sectors including advocacy and policy groups, community-based, faithbased organizations, foundations, media, schools, etc. throughout the Chicagoland area. Data analysis activities are underway, however, results will also be shared regarding characteristics of the communities these organizations serve including:. Age range. Special populations (as defined by the CSTI grant). Underrepresented racial and ethnic communities. DISCUSSION/SIGNIFICANCE OF IMPACT: The Partnership Brokering Tool has provided a format for $\mathrm{CCH}$ to systematically gather information about the relationships staff have cultivated with community groups and organizations. Unlike an email management system, this REDCap project is highly useful in capturing the parameters of our partner pool, identifying partnership gaps, and matching individuals interested in collaborating with researchers or community organizations that have a particular skill set or research interest. The Partnership Brokering Tool has also facilitated stakeholder engagement dedicated to guiding the centers' overall goals, objectives, and programming. Finally, utilizing REDCap has streamlined efforts in reporting quantitative and qualitative data about these organizations. In the next phase of this project, $\mathrm{CCH}$ will utilize the database to assess the nature of the relationship between $\mathrm{CCH}$ and community groups and organizations.
Developing Relevant Community Engagement Metrics to Evaluate Engagement Support and Outcomes

Grisel M. Robles-Schrader ${ }^{1}$, Keith A Herzog and Josefina Serrato

${ }^{1}$ Northwestern University

OBJECTIVES/SPECIFIC AIMS: The goals in this project were two-fold: Develop metrics that assessed community engagement support the center provides, and. Systematically document the fluid and time-intensive nature of providing community engaged research support, as well as key outcomes. METHODS/STUDY POPULATION: The CCH utilized REDCap software in combination with Excel, to create and implement a data collection system to monitor and report on the full spectrum of engagement activities offered by the center. Center staff collaborated in identifying relevant metrics, developing the data collection instruments, and beta-testing instruments with real examples. This facilitated the integration of contextual factors (defined as factors such as the history, size, and diversity of the community, the organizational mission, the structure and size of the CE team, the number of years a university has been supporting community-engaged research work, etc.). Taking a collaborative approach in developing the center's evaluation plan offered the added benefit of facilitating staff/faculty buy-in, building staff capacity, and engaging the team in understanding concepts related to performance measurement versus management. RESULTS/ ANTICIPATED RESULTS: Key benefits of these engagement tracking systems include: consolidating data into a central location, standardizing tracking processes and critical definitions, and supporting more automated reporting systems (e.g., dashboards) that facilitate quality improvement and highlight success stories. Data were compiled and reported via on-line dashboard (REDCap and Tableau) to help center leadership and staff analyze:. Quality improvement issues (How quickly are we responding to a request for support? Are we providing resources that meet the needs of community partners? Academics? Community-academic partnerships?);. Qualitative process analysis (In what research phase are we typically receiving requests for support (e.g. proposal development phase, implementation phase, etc.)? What types of projects are applying for seed grants? After the seed grant ends, are the community-academic partnerships continuing to partner on research activities?);. Outcomes (Are new partnerships stemming from our support? Are supported research projects leading to new policies, practices, programs?). DISCUSSION/SIGNIFICANCE OF IMPACT: There is a gap in the literature regarding meaningful, actionable, and feasible community engaged metrics that capture critical processes and outcomes. This project identified many more relevant metrics and demonstrates that it is worthwhile to take a collaborative, inclusive approach to identifying, tracking, and reporting on key process and outcome metrics in order to convey a more comprehensive picture of community engagement activities and to inform continuous improvement efforts. Community engagement centers across CTSIs offer a similar range of programs and services. At the same time, much of the community-engaged research literature describes metrics related to community-academic grant submissions, funds awarded, and peer-reviewed publications. Experts that work in the arena of providing community engagement support recognize that these metrics are sufficient in understanding the spectrum of engagement opportunities. Community engagement (CE) teams nationally can utilize these metrics in developing their evaluation infrastructure. At the national level, NCATS can utilize the metrics for CE common metrics related to these programs and services. Critical to this 
process:. Leveraging resources that will facilitate collecting generalizable data (national metrics) while allowing sites to continue collecting nuanced data (local programs and services). Gathering input from $\mathrm{CE}$ teams, stakeholders, and researchers to further refine these metrics and data collection methods. Utilizing REDCap, Tableau and other resources that can facilitate data collection and analysis efforts.

3298

Diabetes Prevalence in Rural and Urban Patients Seeking Care from the Veterans Health Administration (VHA): 2007-2012

Danira Medunjanin ${ }^{1}$, Melanie L. Davis, Barbara Wojciechowski, Cheryl P. Lynch, Clara E. Dismuke, Brian Neelon, Neal Axon and Kelly J. Hunt

${ }^{1}$ Medical University of South Carolina

OBJECTIVES/SPECIFIC AIMS: To examine rural-urban disparities in prevalence of diagnosed diabetes in veterans receiving care at the VA and to determine the extent to which demographic factors and obesity levels contribute to identified disparities. METHODS/ STUDY POPULATION: A retrospective serial cross-sectional analysis was employed. A stratified weighted random sample of veterans who received care at a VA facility was selected each year for 2007 through 2012. Rural Urban Commuting Area (RUCA) codes were based on resident zip code. Diabetes was defined by two or more primary or secondary ICD-9 codes for diabetes (250.xx) within a 12 month period. Data were analyzed using complex survey-specific procedures. RESULTS/ANTICIPATED RESULTS: Diabetes prevalence 2007-2012 was lowest in urban (20.5\%-21.0\%), followed by highly rural (21.1\%-22.1\%) and rural (22.3\%-23.0\%) areas with the prevalence being significantly higher on the insular islands (31.0\%-32.4\%). In 2012, $41 \%$ of urban, $43 \%$ of rural and highly rural and $30 \%$ of insular island veterans were obese. Relative to urban areas, the odds ratio for prevalent diabetes was 1.10 (95\% CI: 1.08 , 1.12) for rural veterans, 1.19 (95\% CI: 1.16, 1.23) for insular island veterans, and 1.00 (95\% CI: $0.98,1.02)$ for highly rural veterans. DISCUSSION/SIGNIFICANCE OF IMPACT: Prevalence of diagnosed diabetes is high in veterans residing in rural, highly rural and urban areas, but markedly higher on the insular islands. Understanding the burden of disease and factors driving disparities provides information required to develop targeted interventions.

3015

Enhancing Outcomes in Childcare Settings for Young Children with Behavior Disorders: An Examination of Conscious Discipline Implementation

Sufna Gheyara John ${ }^{1}$, Nicola Edge, Michael Cucciare and Nicholas Long

${ }^{1}$ University of Arkansas Translational Research Institute

OBJECTIVES/SPECIFIC AIMS: 1. Identify the extent of CD implementation for trained childcare teachers. 2. Explore teacher perspectives on the impact of CD. 3. Explore teacher perspectives on barriers and facilitators to full implementation of CD. METHODS/STUDY POPULATION: We conducted a survey with 267 childcare teachers who had been trained in CD across the state, representing early childhood educational environments in urban and rural settings. Specific questions were asked related to level of CD implementation, perceived benefit, and facilitators/barriers to full implementation.
A random subset of the sample (8 teachers) participated in a subsequent focus group to explore survey themes in greater depth. Focus group members were asked about their rationale for attending $\mathrm{CD}$ training, $\mathrm{CD}$ implementation (including barriers/facilitators to full implementation), and perceived impact on their classrooms. The focus group was recorded and transcribed to capture questions and comments. RESULTS/ANTICIPATED RESULTS: Objective 1: 1. $30 \%$ of teachers reported full implementation of CD. 2. $50 \%$ of teachers reported partial implementation of CD. Objective 2: 1. The vast majority of teachers $(95 \%)$ agreed that CD had a positive impact on their classroom, including better structure and enhanced relationships with the children. 2 . The vast majority of teachers (85\%) agreed that CD had a positive impact on the children in their classroom, including increases in problem-solving abilities and selfcontrol. Objective 3: 1 . Most teachers (71\%) reported experiencing barriers to CD implementation, with the majority of those surveyed (93\%) stating that additional implementation support would be helpful. 2. The top three barriers to implementation elicited in survey and focus groups included uncertainty regarding how to begin implementing CD in the classroom, lacking materials for CD implementation, and lacking time to focus on applying knowledge from training into the classroom. 3. The top three facilitators for implementation elicited in survey and focus groups included coaching support for teachers, training agency leadership in $\mathrm{CD}$, and greater perceived impact of CD. DISCUSSION/SIGNIFICANCE OF IMPACT: Childhood disruptive behaviors are among the most frequent reasons for referral to specialized services in and out of the classroom (Sukhodolsky, Smith, McCauley, Ibrahim, \& Piasecka, 2016). Disruptive and aggressive behaviors are problematic, not only for victims of children who are aggressive but also for aggressive children as they age. Although effective treatments exist, the level of effective implementation of these interventions are understudied. These results demonstrate that $2 / 3$ of teachers trained in CD are not fully implementing the model and provides concrete barriers and facilitators to current implementation. These data will provide the initial foundation for the development of a targeted implementation strategy that supports full implementation of CD within early childhood education settings.

3484

\author{
Examining the Use of Mobile Technology Among \\ Low-Income African Americans and Hispanics with \\ Hypertension \\ Emily Kostek ${ }^{1}$ and Yendelela Cuffee \\ ${ }^{1}$ Florida Department of Health
}

OBJECTIVES/SPECIFIC AIMS: The primary objective of this study was to assess the interest in using mobile technology to manage hypertension and assessing medication adherence among African Americans and Hispanics living in a low-income urban community in Central PA. METHODS/STUDY POPULATION: This qualitative research study was designed using structured interviews with 30 African American participants with hypertension. The study survey consisted of 43 questions which included demographics, use of technology, technology literacy, health literacy, and medication adherence. Participants self-reported their responses about use of technology, literacy, and adherence. The data was examined using summary statistics. RESULTS/ANTICIPATED RESULTS: Out of 30 participants, $61 \%$ identified as African American and 30\% identified as Hispanic. Overall, participants reported high use of 